\title{
On an integrated analytical approach to describe quality design process in light of deterministic information theory
}

\author{
Tamer El-Khouly and Alan Penn \\ University College London, UK
}

\begin{abstract}
This paper introduces a methodology to analyse design linkographs by quantifying entropy at each single move throughout the design process. The method adopts the deterministic information theory proposed by Titchener [1], [2], [3] to develop a quantitative model aiming to highlight the significant nodes by coding the dependency relations (backlinks and forelinks) into character strings of information. Two computational methods are suggested to quantify $T$-code sets on a microlevel at every single utterance. This proposition is intended to capture repetition of patterns and hierarchy in the linkograph pattern. This quantitative approach is integrated with a qualitative model of judging sketching episodes and evaluating the relations between the instantaneously evolved products during the design process such as the interim sketches. The results point at significant correlations between quantitative and qualitative models on the key nodes to occur in the process to identify the emergence of novel ideas and describe design creativity.
\end{abstract}

\section{Introduction}

A linkograph is a representation that traces the associations of every single move (utterance), the design process can be looked at as a linkograph pattern that displays the structure of the design reasoning. The venues of dense interrelations are overtly highlighted on the graph as well as shallow ones and can be further interpreted through the emerging artefacts (interim sketches etc.) along the process. To reveal the structure of design reasoning, the proposed analytical model builds on the hypothesis that the emergence of ideas is an outcome of the interplay between reflection in the mind and the alternative solutions generated. 
The aim of this proposition is to discover how to correlate a quantitative method to signify pivotal moments or key venues in the development of ideas with the corresponding cognitive activities, foremost of which are externalised sketches and drawings.

The prime goal of an objective tool to quantify the dependency relations between design units in a linkograph is to describe the characteristics of a design idea and its hierarchical structure; and to develop a qualitative model that describes a designing episode and identifies starting and ending positions of reflections between the mind and the media of representation.

The multi-level complexity of cognitive process and syntheses of designing are the primary motivations for the development of an integrated analytical approach to understand the nature of the design process and its products.

Relations in linkography are looked at from two perspectives, information and entropy. We propose that relations can be transcribed into a character string of information coding dependencies into binary symbols.

Deterministic information theory proposes a set of codes where each code measures a parameter on the character string of information. Titchener developed this theory in stages. T-code sets comprise two primary algorithms: T-decomposition decomposes the complexity of the character string into its possible primary level; in contrast, $T$-augmentation augments the primary units' "codewords" to reproduce the full character string. By using this theory we can compute the T-code measures such as T-entropy, T-complexity and T-information for any string. [1], [2], [3].

\section{The design process as a multi-level complex system}

This study begins by highlighting the characteristics of linkographs, which have been inferred through a series of analyses on different patterns of case studies. The following structure forms the hierarchy of linkograph: nodes, clusters and networks.

Segregation or integration of networks varies from case to case: the pattern is sometimes coherent and parts are connected despite the diversity of the cognitive activities undertaken, but this cannot be postulated as a general rule because sometimes a total separation occurs between two or more subsets. Based on this, the structure of linkograph varies between fully connected and saturated or totally random and disordered. Both are extreme situations in design thinking. Thus three prototypes of linkographic patterns are categorised: highly ordered, structured and disordered, reflecting integration, coherence and diversification respectively. 

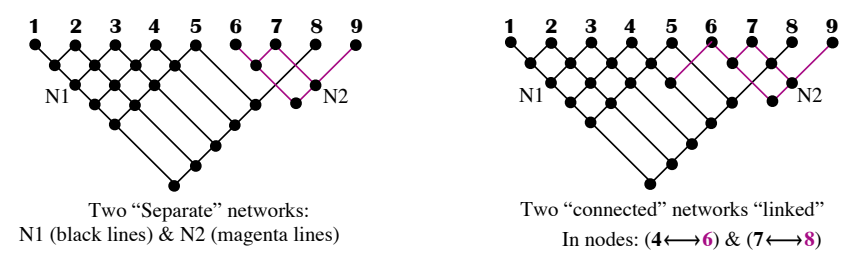

Fig. 1 The relations between networks in a linkograph; separated or connected

The linkograph reflects a state of design that changes through time. This change might underlie an entire state with properties that cannot be identified through the outward appearance of the pattern. Schön [4] suggests that a design transforms its state according to the change of repertoires in the mind. The challenge of this paper is to understand how the transformation of ideas from one state to another can be captured. In addition, the reflective practice (a methodological design paradigm developed by Schön [4]) plays a vital role in the manipulation process of the artefacts of design. Why do certain interim artefacts not reflect exactly what was happening in the designer's mind at a particular moment? Although the sketches result from the mind, there will be instances when the sketch will reflect back to some (buried) insight in the mind.

Goldschmidt [5] revealed that a designer does not represent images held in the mind, as is often the case in sketching by non-designers, but creates visual displays that help induce images of the entity that is still being designed. This is considered an intermediate medium of representation to mediate between mental manifestations and the design outcomes.

In relating the cognitive processes with the linkograph, various patterns of mental representation can be inferred from studying the relations that can be made between the units of design. Fig. 2 demonstrates how a linkograph can be configured from ordered to disordered patterns.

What deserves attention is how the design process is built up from the parts to the whole in order to look at the venues of high creativity within the structure. The proposition is therefore to investigate the synthesis of relations in every action and globally to understand the structure and describe the design process. In this context, it is vital to distinguish between information and entropy since most of the current publications on protocol analysis adopt entropy as a central element to describe the design process. 


\begin{tabular}{|c|c|c|}
\hline $\begin{array}{l}\text { Pattern } \\
\text { Type }\end{array}$ & Description of the State & Linkographic Configuration \\
\hline Order & $\begin{array}{l}\text { - A highly ordered pattern states an ongoing } \\
\text { identical probability to move from one epi- } \\
\text { sode to another } \\
\text { - It reflects a state that a designer keeps per- } \\
\text { forming the same actions ever then } \\
\text { - A pre-mature fixation effect of a certain idea } \\
\text { may occur }\end{array}$ & 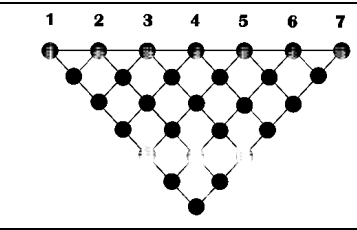 \\
\hline Structure & $\begin{array}{l}\text { - A structured pattern delivers variable chan- } \\
\text { ces to develop an idea from one single utter- } \\
\text { ance to another } \\
\text { - A diversification of various design ideas is } \\
\text { experienced in the process }\end{array}$ & $\begin{array}{llllll}1 & 2 & 3 & 4 & 5 & 6 \\
9 & 9 & 9 & 9 & 9 & 0\end{array}$ \\
\hline Disorder & $\begin{array}{l}\text { - A chaotic design situation reflects unrelated } \\
\text { thoughts on the design situation } \\
\text { - It might cause total confusion and loss on } \\
\text { the track of development. }\end{array}$ & $\begin{array}{llllll}1 & 2 & 3 & 4 & 5 & 6 \\
0 & 0 & 0 & 0\end{array}$ \\
\hline
\end{tabular}

Fig. 2 Different states of design reflect different patterns

\section{Information and entropy}

Information and entropy are two angles from which to look at linkography. While the information theorist looks at the probability that can be created for a sequence of relations for a single item, the entropy theorist considers the set (which is made up of items) a microstate on its own for the system. The two theories are in opposition. Entropy grows with probability, while information increases with improbability. The less likely an event is to happen, the more information its occurrence provides.

Entropy is a measure of the state of disorder for any system. The aim of estimating entropy in information theory is to predict the probability of an event occurring. The objective of information theory is to investigate probability by establishing the number of possible sequences that can be created per single item. The sequence of an item is not taken into account in entropy theory but is necessary in information theory. Information theory is adopted to develop a quantitative approach to quantify the possible relations that are likely to occur at each item in the linkograph. Table 1 highlights the differences between information and entropy. 
Table 1 The differences between information and entropy

\begin{tabular}{lll}
\hline Information Theory & \multicolumn{1}{l}{ Entropy Theory } \\
\hline Structure & $\begin{array}{l}\text { Nothing is better than those certain "se- } \\
\text { quences" of items that can be expected } \\
\text { to occur }\end{array}$ & $\begin{array}{l}\text { Sets constitute the main characteristics of } \\
\text { the structure }\end{array}$ \\
\hline $\begin{array}{l}\text { Is about "sequences" and "arrange- } \\
\text { ments" of items }\end{array}$ & $\begin{array}{l}\text { Is about the "overall distribution" of kinds } \\
\text { of item in a given arrangement }\end{array}$ \\
$\begin{array}{l}\text { The less predictable the sequence, the } \\
\text { more information the sequence will } \\
\text { yield, and the more remote its represen- } \\
\text { tation from order }\end{array}$ & $\begin{array}{l}\text { The more remote the arrangement of sets is } \\
\text { from a random distribution, the lower its en- } \\
\text { tropy, and the higher its order representation }\end{array}$ \\
$\begin{array}{l}\text { A highly randomised sequence will be } \\
\text { said to carry much information by the } \\
\text { information theorist because information } \\
\text { in this sense is concerned with the } \\
\text { probability of this particular sequence }\end{array}$ & $\begin{array}{l}\text { A randomised distribution will be called by } \\
\text { the entropy theorist "highly probable" and } \\
\text { therefore of low order because innumerable } \\
\text { distributions of this kind can occur }\end{array}$ \\
\hline
\end{tabular}

\section{Towards an integrated model to describe the design process}

There are various models that aim to study the design process. This paper explores two challenges: how to develop a quantitative tool to quantify the dependency relations in the linkograph and how to judge the dependencies in a qualitative way. This bi-modal methodology obtains insight from the application of deterministic information theory [1], [2], [3] and the paradigm of reflective practice process [4]. The aim is to provide an integrated approach to identify the formation of design ideas.

\section{The quantitative model}

\section{Methods to quantify linkograph via Shannon entropy}

Shannon entropy aims to measure information associated with a communication source. Shannon suggested that the amount of information carried by a message is based on the probability of its occurrence [6]. The application of Shannon entropy to linkography, first proposed by Kan and Gero [7], aims to measure the degree of "probability" and "surprise" of the emergence of ideas in the design process, with a hypothesis that high uncertainty motivates the designer to explore the design space for more solutions (i.e. to become more creative).

The application of classical entropy to quantify linkograph has been argued by Shyan-Bin Chou [8]. To rectify the estimation process of Shannon 
entropy, his method adjusts entropy value with a pattern-matching factor to pick up the frequency of appearance of patterns into the estimation.

We argue that the application of Shannon entropy treats the linkograph in terms of sets and networks regardless of the sequential arrangement of occurrence of microelements that constitute the set according to time (the order of relations is according to the emergence of nodes). It looks at the overall distribution of sets in a global manner. Fig. 3 shows two different linkographs that contain the same number of nodes (same $n$ size of the system) but have different distribution. Both graphs sum up the same value of linked and unlinked relations giving an identical single entropy value:
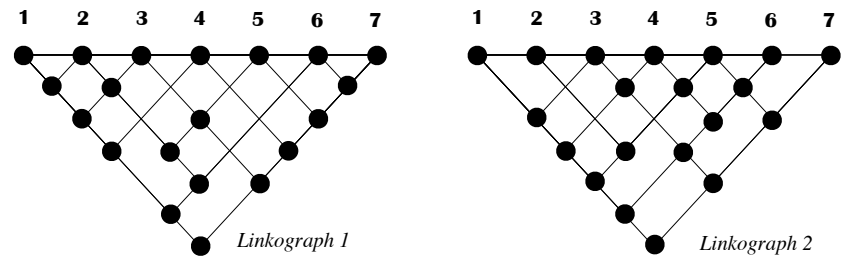

Processing Shannon Entropy:

$\left[\mathrm{H}=-\left(p_{\text {linked }} \cdot \log _{2} p_{\text {linked }}\right)+\left(p_{\text {unlinked }} \cdot \log _{2} p_{\text {unlinked }}\right)\right]$

The total number of possible relationships $=n[(n-1) / 2]=7(6 / 2)=21$

Where $\mathrm{n}$ is the total size of the linkography (the number of nodes)

The total number of "linked" relations in both graphs is $=13 \rightarrow \simeq 61.9 \%$

The total number of "unlinked" relations in both graphs is $=8 \rightarrow \simeq 38.1 \%$

$\mathbf{H}=-\left[(13 / 21) \times\left(\log _{2}(13 / 21)\right]+\left[(8 / 21) \times\left(\log _{2}(8 / 21)\right]=\mathbf{0 . 2}\right.\right.$ bit/bits

Fig. 3 Two different linkographs give identical values of Shannon entropy

As linkographs with different arrangements of relations reflect different design processes, the classical application of entropy is only associated with the quantity of links regardless of variation in distribution. The paradox still exists if we refer entropy into a single node - can we address the right distribution of the created relations on each node in the linkograph? And, can we reduce such a multi-level complex system to one single value?

A synopsis on the deterministic information theory [DIT]

Titchener first presented the DI theory in 1998. A series of developments followed to obtain more accurate computation on variable-length strings of codes [1], [2], [3]. This theory presents $T$-code sets to encode the frequently occurring symbols of an information source, in which messages from any source can be coded into alphabets or symbols of codewords forming a character string of information.

Two coding alphabets can be characterised when coding the dependency relations in the linkograph: ' 1 ' for linked and ' 0 ' for unlinked. In this method, the linkograph can be transcribed into an alphabetical string of the 
two symbols and with a string length of all the relations that can be coded from a node to the others (length $=n-1$; where $n$ is the system's size; the number of all moves in the linkograph). To code information at each design move, we look at its preceding and following relations and thus we can extract a character string and inspect its properties. As a rule of thumb, prior to DIT, however, characters cannot be processed directly without forming what is often called in the theory, "codewords".

T-code sets form or decode the codewords to reach an unambiguous serial transmission of information. A T-code set has two properties: it augments symbols to produce the codewords to construct the string, or decomposes the string to the microelements required to build it. In the augmentation process, $T$-augmentation can be seen as a string production algorithm, while $T$-decomposition may be seen as an algorithm that removes codeword boundaries in a hierarchical order. T-codes process the string by parsing the codewords in "forward" and "reverse" directions to compute its complexity, information content, and entropy providing three measures: T-complexity, T-information and T-entropy. The advantage of this method distinguishes different patterns of codewords and accounts for the position of each symbol in the string. The repeating appearance of codes, such as 000000000 or 111111111 is considered an extreme measure of the least uncertainty of serially communicated information.

Consider the following bits strings: 00000000001111111111 and 01001101100010101110. Despite containing a balanced number of 1s and 0s (giving the same Shannon entropy value), both strings deliver different T-code sets. Yet to the casual observer, it is clear that the second string contains a little more information than the first due to the complexity added by the differentiation of arrangement of symbols. In this case, a clear difference is shown if T-code method is processed on each string.

At the onset, DIT is introduced to this research area to emphasise the difference between classical entropy and T-codes. Shannon entropy assumes that a string is made up of $n$-grams (n-bit substrings) while T-codes assume that the string is a codeword from some sets and measures the number of weighted steps required to build that code [9], [10]. T-entropy looks at codewords while Shannon entropy looks at $n$-grams or $n$-bits. This method delivers accurate estimation of entropy that accounts for the arrangement of patterns of codewords at the level of micro items rather than classical entropy in the linkograph. It considers the position of any symbol in the string in the computation process which is crucially relevant to the context of sequential emergence and occurrence of moves in the design process. The following equations are principal to DIT: 
T-complexity is: $\mathrm{C}_{\mathrm{T}}(\mathrm{x})=\sum_{\mathrm{i}=1} \log _{2}\left(\mathrm{k}_{\mathrm{i}}+1\right) \ldots$ (I)

Where $k$ gives the number of adjacent copies of the T-prefix found at the position in the string when the T-prefix is identified for the first time.

T-information is: $\mathrm{I}_{\mathrm{T}}(\mathrm{x})=\mathrm{li}^{-1}\left(\mathrm{C}_{\mathrm{T}}(\mathrm{x})\right) \ldots$ (II)

Where $i^{-1}$ is the inverse logarithmic integral

T-entropy is: $\mathrm{H}_{\mathrm{T}}(\mathrm{x})=\mathrm{I}_{\mathrm{T}}(\mathrm{x}) /\|\mathrm{x}\| \ldots$ (III)

Generally, the richer the variety of symbolic arrangements, the more information the string carries. T-entropy predicts the patterns occurring in the string, where a high value indicates minimal repetition of patterns and means new patterns are appearing in the string with high unpredictability. A low T-entropy indicates a high repetition in the string - highly predictable information [2], [9], [10], [11].

T-complexity and T-entropy tend to "converge" as the string gets longer; the effect will be seen mostly with longer strings (hundreds or thousands of bits). Lack of accuracy increases with strings of less than 25 characters.

\section{Quantifying the linkograph via T-code sets}

This section introduces a computational tool to process the linkograph. Two methods are proposed: one operates on the level of individual nodes; the other subdivides the graph into subsets or sub-linkographs.

Method 1: Processing T-codes for individual moves. Our prime target is to compute $T$-complexity and $T$-entropy at each node in the linkograph, where both measures fluctuate throughout. In this method, the process to compute extracted strings can be carried out via one of three ways, which differ according to the direction of reading links (backward or forward):

1. Extracting only backlinks string per each node: In this method, all relations are extracted in a reverse way to the emergence (from end to start). For example: node 5 has relations (linked or unlinked) with 4 , 3,2 and 1, but 1 has no back relation since it is the starting point.

2. Extracting only forelinks string per each node: In this method, all relations are extracted in a forward way, like the direction of growth in the linkograph (from start to end). For example: node 1 has forward relations (linked or unlinked) with $2,3,4,5, \ldots \mathrm{n}$, but $\mathrm{n}$ has no forward relations since it is the end point. Methods 1 and 2 are both synchronous to the emergence of links. See Figs. 4-a and 4-b to check the method per each reading direction.

3. Concatenating backlinks and forelinks together per each node: This is a third method based on concatenating both strings (backlinks and 
forelinks) per each node together in order to process one longer string at once (see Fig. 5).

Many methods can be suggested to extract a character string of information. The proposed method for a linkograph is to undertake the synchronous occurrence of nodes and to consider the direction of reading the relations. Fig. 4-a illustrates an application of "reverse" or "forward" methods of processing strings on a linkograph where the direction of reading makes a significant difference to the final results.

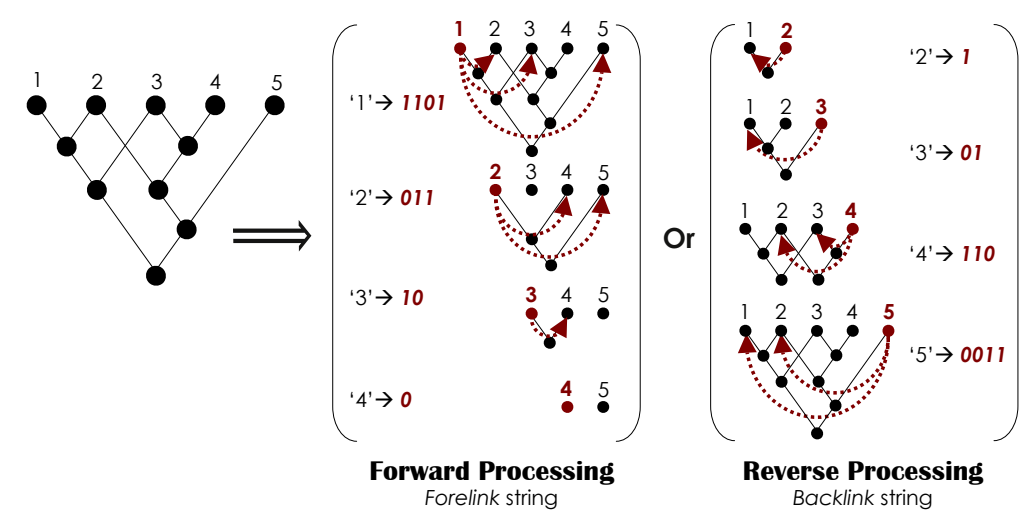

Fig. 4-a An application of extracting the strings: forward or reverse

For example, in a linkograph with 5 nodes, if strings are extracted in reverse, node ' 1 ' has no preceding relations, ' 2 ' might have a relation with ' 1 ', ' 3 ' has two probabilities with ' 2 ' and ' 1 ', ' 5 ' has four with all the preceding nodes. Generally the string out of ' $n$ ' has (n-1) string size, but in forward processing, the string out of ' 1 ' will have four probabilities while the last point ' 5 ' will have no probabilities with any following nodes.

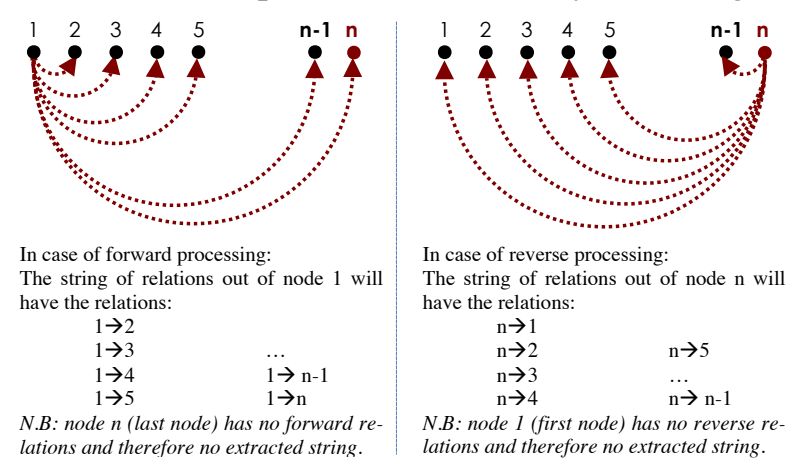

Fig. 4-b Extracting the string in two directions: forward or reverse 


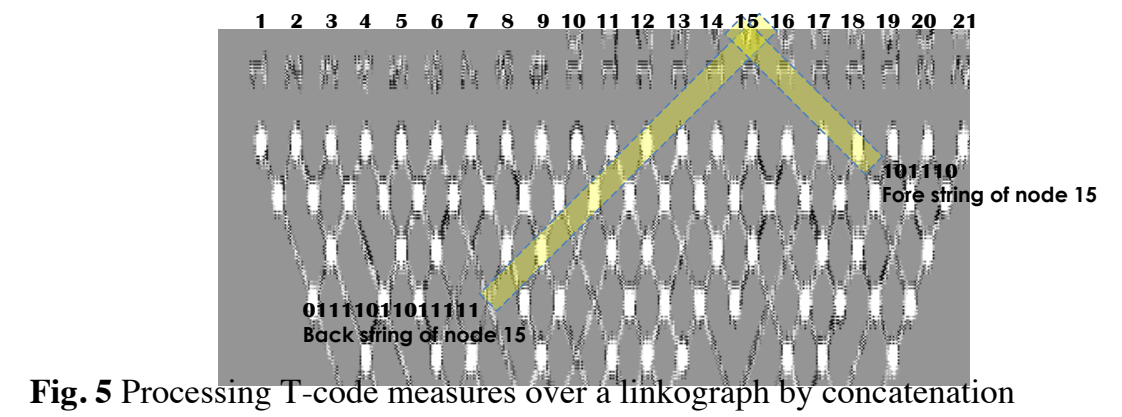

In this hypothetical graph, node (15) has the following coded relations: Backlink string is 01111011011111 and Forelink string is 101110 and the concatenation is 01111011011111.101110.

Method 2: Processing T-codes for subsets or sub-linkographs. In this method, the linkograph is subdivided into a series of subgraphs. The subdivision can be made in two ways: time rate or amount of nodes. In each, back and fore strings can be computed similarly to method 1 (individual or concatenation). However, it should be noted that measures per frame must be normalised to the n-size of the sub-system in order to "relativise" the results of the subgraphs together. This is necessary to achieve the required accuracy but the result is conditional on being divided by the logarithm of the $\mathrm{n}$ - total number of nodes in each subset $\left(\log _{2} \mathrm{n}\right)$. The calculation process starts by setting up the number of nodes (or occurrence rate) in a hypothetical window that slides across the baseline of the linkograph. The more a window displaces, the more nodes are included in the estimation process.

This method is built on the basis of two factors - time and activity - that must be described in the design process. We can inspect a certain activity that is relevant to time of emergence; such as looking at a certain sketch (design medium) that has specific start and end points. The emergence of action and the formation of concept are illuminated to find which actions are pivotally responsible for the emergence of a novel idea. The application of T-codes to linkography is looked at in a design case study in Sec. 4.

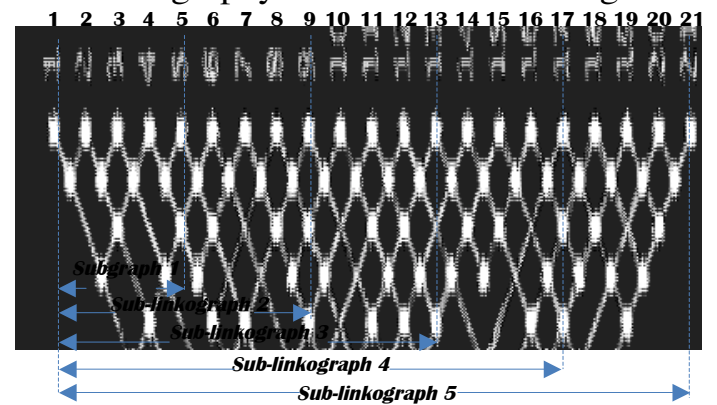

Fig. 6 Processing the linkograph as a series of subgraphs 


\section{Qualitative judgements on sketching episodes}

The purpose of this approach is twofold. First, it sets up the starting and ending of what is often called a design move or utterance. Second, it aims to unveil cognitive mutual reflections with instantaneously externalised design artefacts all through various modes of representation (artefacts are the interim products such as sketches, 3D models etc.). As such, we aim to illuminate stimuli responses with respect to the sketching episodes; how they help the designer to break away from a frame of reference (which may lead to fixation) to proceed to a new one. The linkograph can then be drawn according to the judgements of dependency relations.

While cross reflection is an imperative key to understand the sketching interoperability with the mind, instantaneous perception is also a design process included in judging the sketching episodes. Tschimmel [12] suggests design as a perception-in-action process has five nonlinear intersected procedures: the perception of the task, the perception of a new perspective, the perception of new semantic combinations, the perception in prototyping, and the perception of users' reactions.

Hence, we define a sketching episode as a transformation in perception from one state to another while marking out the drawings prior to the design situation and to the interim reflection with the sketch still in progress.

Any sign that the designer has perceived a notion to break out of a frame of reference and shifted to another is considered an insight according to Akin and Akin's description [13]. A creative insight moves the perception completely to a different state that is independent from the current design situation. The design moves are hence coded based on two sets of contribution: actions preserve continuous reflections in the mind and actions defy continuous reflections.

Preserving reflection proceeds on the initial concept. It takes various forms of activity, such as replication, redefinition or advanced incrementation, in the same design state. Defying reflection introduces a new item to the current state. It has a different taxonomy of actions that operate to change the design situation, e.g. divergence, synthesis and reconstruction.

Sketching is an act to perceive and reflect cognitive actions since it plays a central role in transferring notions in the mind into a design configuration. Goldschmidt [14] stated two types of sketching: type (1) aims to transform imagery into new forms of combinations and is considered as rational mode of reasoning; type (2) is sketching to generate new imagery of forms in the mind and is a non-rational form of design thinking. Our proposition primarily adopts all the preceding elements into developing a qualitative model to assess sketching episodes (see Fig.7). 


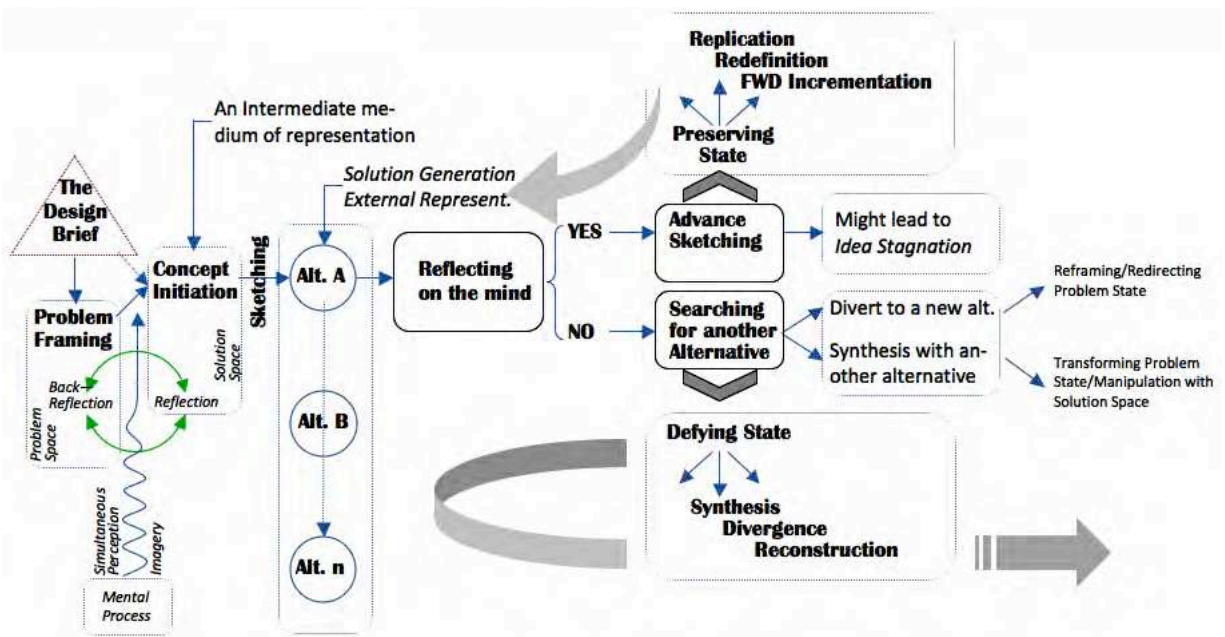

Fig. 7 Qualitative judgements model to describe sketching episodes

\section{An empirical case study on architectural design process (the} phase of earlier initiation of conceptual ideas)

We look first at the brief given to the designer, a chartered architect with 12 years' experience, in a design experiment at Bartlett School of Graduate Studies. The compatibility between the quantitative and qualitative results is in our attention to assess the adequacy of this model.

\section{The design brief}

The designer was asked to design a pavilion at Expo-Shanghai, 2010, presenting an image of her country from her own perception. The brief was left open-ended with no specific requirements or constraints to give the designer free rein. The conceptual idea was to be presented via any means of representation without any specific drawings or projections being requested and with no intrusion from the researcher. The process was videorecorded and the designer was asked to retrospectively explain the design idea for the serial order of three A2-size sketches produced in this session.

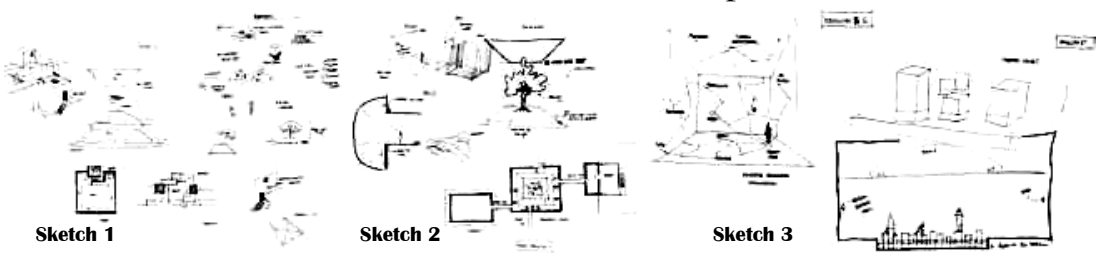

Fig. 8 Snapshots of sketches from the design experiment 


\section{Describing the process, products, and significant moves}

The process began by setting up elements to initiate the concept. These elements were considered to reflect the nature of Greece, where the designer's country. Eight elements were drawn in a diagrammatic form: (1) Sea ripples: Circulation in and around lakes; (2) Built environment: boxes and light; (3) Complexity: steps, organic or orthogonal forms (4) Sun and sky; (5) Olive and lemon trees; (6) Colour scheme (blue, white, turquoise) (7) Rock and water; and (8) Strong shadows.

Five independent pavilions were grouped in one site plan, each reflecting an element from the preliminary set (sometimes a synthesis of some elements together). The aim was to reflect impressions and spatial experiences to the users. Pavilions 1, 2 and 5 strongly relate to the preliminary set of design elements; the 'Stepped Forms', 'Olive Tree', and 'Rocks and Water'. Pavilions 3 and 4, 'The Tunnel' and 'Links and Cities', are considered as "creative leaps" on the flow of idea development. They have no strong links with the predecessors or even with the last pavilion (Rocks and Water). In spite of being produced serially pavilions 3 and 4 have no relations and their concepts are totally independent. In retrospective comments, the designer described those products came up while designing.

\section{Transcription and analyses}

The dependencies between design utterances are examined and coded according to the order of occurrence of interim artefacts (sketches). The designer wanted to implement natural lighting as a central concept. Artefacts 2 and 3 and the last slide (pavilions 1, 2, and 5) adopt roof skylights. Tracking this idea from one pavilion to another helps us to perceive the lateral transformation beyond the interim artefacts. The investigation was made on an overview of the whole but also on pair-wise comparisons. The sudden "absence" and "appearance" of the skylight element encourages further investigation of the reasons beyond this and search into the possibility that creative insights might occur in parallel to the discourse.

Activities at points (52), (71) and (79) in the linkograph need consideration since no direct reflection is perceived on these particular moves. The sudden occurrence of node (55) is a change of state, when the designer diverted her train of thought from designing the olive tree pavilion to designing a new one. The tunnel pavilion is a spectroscopy on various colours and a gradual diffusion of artificial light. However, with lighting as a prime concept, the outcome is obviously different since the form and installation drawn for this pavilion are for various kinds of artificial lighting.

Point (71) is a paradigm shift, probably divergent and more than a mere change of state as in action (55). At this point, the designer suddenly di- 
verted to draw a new element on the dispersion of Greek community around the world. The illustration of this idea, in pavilion 4, shows names of cities to which Greeks used to immigrate: Melbourne, London, Istanbul, etc. The designer called this pavilion "Interactive Installation Immigration". The names of cities are symbolised by dispersed nodes in a cubic form and connected with links. Colours and lights are embedded in ways that are different from in the preceding pavilions. The idea has intruded into the prevailing flow in this way.

Point (24) is a back-reflection (sketching back) to reframe the idea of composition and complexity in the preliminary set of elements. The designer drew a diagram of overlapping terraces and masses around a central atrium to assemble a parti (conceptual artwork) of the independent pavilions after designing the stepped form pavilion (nodes 17-23). Point (52) is also a back-reflection about irrational openings and balconies. The designer went back over this diagram to add to the first set of elements after designing the 2D-plan of the Olive Tree pavilion (nodes 31 to 51).

Table 2 The integration of qualitative and quantitative approaches in one model

\begin{tabular}{|c|c|c|}
\hline$\#$ & Phase & Detail \\
\hline 1 & Transcription & Transcribing the design activities accordingly to the time of occurrence \\
\hline 2 & Interim artefacts & $\begin{array}{l}\text { Setting out the interim outcomes in the order of emergence (design arte- } \\
\text { facts, sketches, etc.) }\end{array}$ \\
\hline 3 & $\begin{array}{l}\text { Identification of } \\
\text { design episodes }\end{array}$ & $\begin{array}{l}\text { Processing the qualitative/cognitive model by identifying each design } \\
\text { move according to the notion of "reflection-in-action" }\end{array}$ \\
\hline 4 & Coding process & $\begin{array}{l}\text { Coding the externalised drawings in relation to the pertained cognitive } \\
\text { activities. The dependency relation is looked at through two angles: } \\
\text { 1) The relation between each artefact and the preliminary set of design } \\
\text { elements (the interim artefacts and initial conceptual elements) } \\
\text { 2) Pair-wise comparison between sequential pairs of drawings. This is to } \\
\text { investigate the lateral transformation and search for any sudden insight } \\
\text { that might occur in the prevailing flow }\end{array}$ \\
\hline 5 & Reflection-in-action & $\begin{array}{l}\text { Finding the "reflections" and "back-reflections" amongst the sketches to } \\
\text { classify a hierarchy of the products }\end{array}$ \\
\hline 6 & Linkography & Drawing the linkograph \\
\hline 7 & String computation & $\begin{array}{l}\text { Processing the quantitative model (T-code measures) } \\
\text { - } \text { Setting out a matrix of relations } \\
\text { - } \text { Processing the T-code algorithm }\end{array}$ \\
\hline 8 & Archiography & $\begin{array}{l}\text { Drawing the archiograph (another representation of the linkograph but it } \\
\text { reflects the relations in a clearer way) }\end{array}$ \\
\hline 9 & Comparisons & Comparing the archiograph with the interim artefacts \\
\hline 10 & Correlations & $\begin{array}{l}\text { Checking out if correlations exist between the quantitative model and } \\
\text { qualitative judgements }\end{array}$ \\
\hline
\end{tabular}


To summarise this proposition, coding relations in a linkograph is mainly about tracking reflections with respect to their order of occurrence in the context of the interim artefacts to construct an adequate linkograph. Table 2 presents the procedure and order of analysis in the proposed.
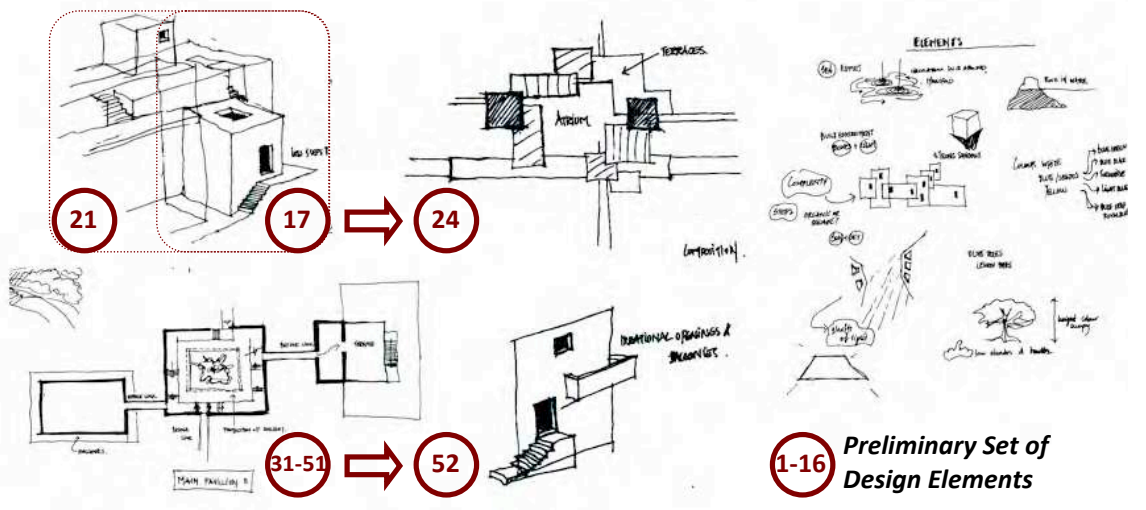

Fig. 9 Snapshots of examples of sketching episodes and idea transformation

\section{Results and discussion}

Starting with the quantitative model (T-code sets), the results of the two proposed methods to compute strings' entropy show the following:

1 . The two methods of computation, directional string or concatenation, help to exemplify the significant nodes in two different situations. The former (directional back string) explains dependency relations on the instantaneous design situation in progress (before completion) and the latter (concatenated string back and fore) explains the design process after the completion of the whole design situation.

2. Correlations and comparisons between results can be achieved from these computational methods because all values are relativised to the "n-size" of the system. This is one of the main characteristics owing to deterministic information theory and T-code sets.

3 . The numeric results provide measures that can be compared in correlation with the interim design artefacts or cognitive activities associated with the relevant moments.

In the whole process, node (53) achieves the highest degree of integration with a value of 6.09. The lowest degree of integration is delivered by node (25) and (71) with a zero value. At node (53), the designer redrew a diagram, titled "irrational openings and balconies", as a new element of 
design to generate new syntheses of form in her mind (that might be obtained again afterwards in the designing discourse).

At node (25), the designer put down the pen and glanced at the design brief. This happened between two sketching episodes: (24) in which she drew a new form into the set of elements "composition of terraces and overlapped masses"; and (26) when she shifted to design the "Olive Tree" pavilion by scribbling a diagram of shrubs, passages and green landscape. Node (25) is a disconnection on the train of thought.

Likewise, node (71) is a rupture in the running activity - sketching episode (70) is about adding quotations on 2D-section-the tunnel pavilion where she drew suddenly a diagram of a new element entitled links on a different sheet. Despite delivering zero connections with preceding actions, point (71) has strong linking relations with following actions until the completion of pavilion 4 .

Subsequently, three levels are denoted to inspect the structure of design hierarchy: (1) the preliminary level of concept initiation (an intermediate medium); (2) a level of continuous forward sketching and externalisation of ideas; and (3) a level of back-reflections to generate imagery of forms in the mind (represented by adding new design elements to the preliminary set of concept initiation). Fig. 10 illustrates the distribution of sketching artefacts (snapshots) across these three levels.

Point (31) achieves the highest T-complexity and T-entropy values via the concatenation method with values of 17.98 and 0.8 . The designer shifted the sketching episode from two different projections (3D perspective to 2D plan). This happened while designing the "olive tree" pavilion in order to further enhance and describe the concept in detail. Exchanging thoughts "back and forth" and switching the idea between different media (drawing projections) is a clear example of the mutual reflection between the design media and the mind (between two cognitive structures - internal and external). The integration value for point (31) is 2.98 , which is the lowest value relative to the whole linkograph. However, (31) reflects a high degree of understanding and developing the idea through different media of representation. In this discourse, integration is inversely correlated with string measures (T-complexity and T-entropy). See the numeric values of some significant nodes in Table 3.

The lowest degree of T-complexity and T-entropy is delivered by node (49) with values 7.25 and 0.21 . At this node, the designer traced over an existing sketch but just with a thicker pen to highlight a plane cutting through the tree pavilion. The utterance is not crucial to the development but an emphasis on the activity. 


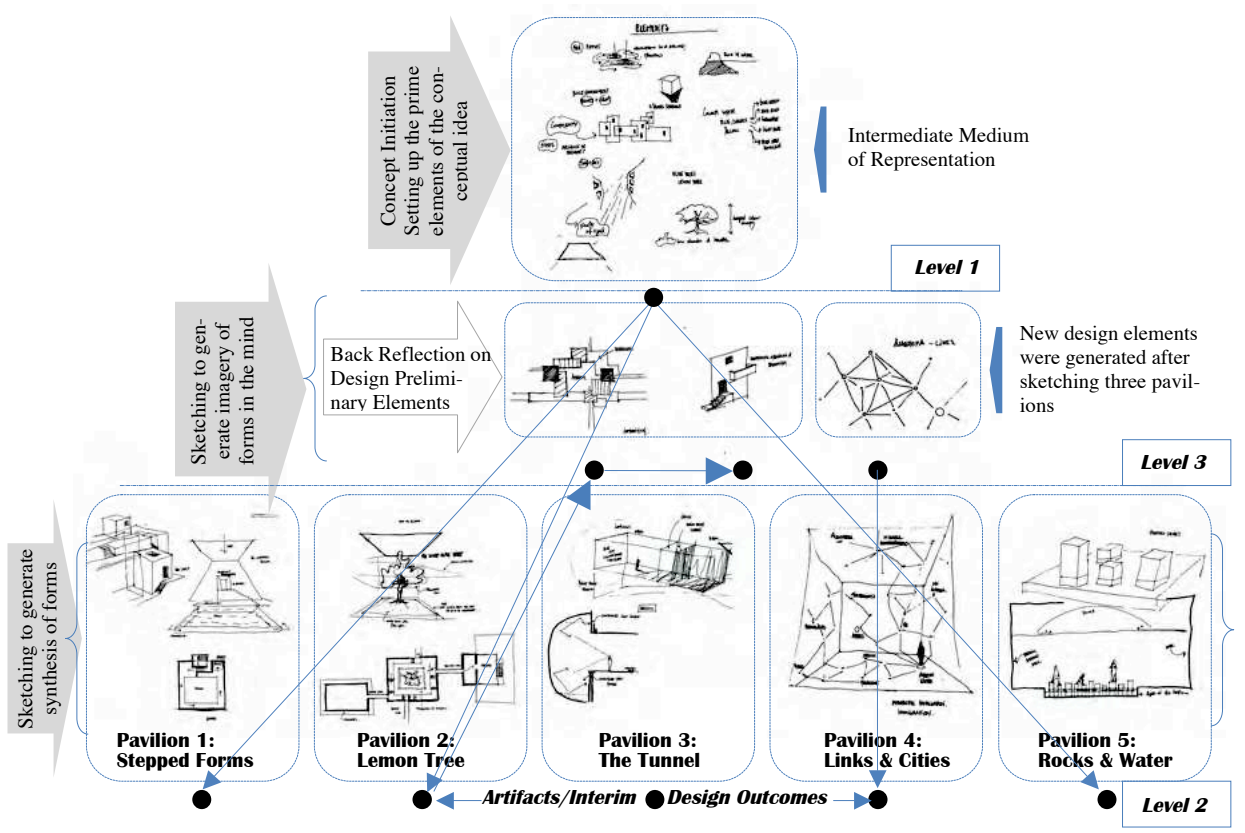

Fig. 10 A hierarchical classification of sketches based on qualitative judgements: (reflections) and (back-reflections)

Figs. 11 (a) and (b) overlay methods of computing strings on the archiograph. A change of state is often observed in integration, T-complexity and T-entropy values on the switches between different media (sketches) on how the idea utterances are synthesised in the process. Archiography is a process to represent dependencies amongst nodes. It was developed to illustrate relations in a way to avoid dense clusters not on the baseline. It looks at graph connectivity rather than clusters of nodes. Archiographer ${ }^{\odot}$ software was developed to build up the relations and draw the archiograph.

Pavilions 3 and 4 are semi-disconnected from the whole linkograph and the relations are similarly distributed amongst nodes. Therefore, no significant fluctuation is seen with T-codes compared to integration values.

The correlation between syntactical measure (integration) and T-code sets (complexity and entropy) are examined in detail in a previous study by the author. El-Khouly and Penn [15] study the correlations on various systemic graphs that share multi-level complex properties such as 'linkographs' and describe the structure of state via different spectrum of patterns; orderliness versus disorderliness. 


\section{In conclusion}

This study outlines a qualitative approach for describing design hierarchy. In this discourse, it discusses the compatibility of merging quantitative and qualitative models. Forming non-rational syntheses serves to introduce new boundaries to the design programme and also encourages the exploration of new functions that have not been explored before. Throughout the proposed model, we can detect a multi-levelled concept that has been conducted through a design process, to see how the design serves it goals and how it sets new goals. A multi-level design concept is evolved through:

1. An intermediate medium of representation for the concept initiation.

2. An execution process of the idea.

3 . A retrospective reflection on earlier thoughts.

The observed complexity of idea transformation is owing to the fact that there are no isolated concepts in ordinary thoughts except those rare but remarkable breakthroughs.

Despite the meaningful interpretations that can be made by implementing the Shannon method, its disadvantage is that it estimates entropy in a global manner since the process is done layer-by-layer. The aspiration of this study is to append another measure to acquire information from a nonlinear system such as linkography. T-code sets provide an objective tool to inspect entropy in a multi complex design process, working in integration with qualitative judgements on the design outcomes.

Table 3 Various quantitative measures of some significant nodes

\begin{tabular}{|c|c|c|c|c|c|c|}
\hline \multirow{2}{*}{$\#$} & \multirow{2}{*}{ Extracted Concatenated Strings } & \multirow{2}{*}{$\begin{array}{l}\text { Integration } \\
\text { Values }\end{array}$} & \multirow{2}{*}{$\begin{array}{l}\text { Dynamic } \\
\text { Backlink } \\
\text { Entropy }\end{array}$} & \multicolumn{3}{|c|}{ String Computation } \\
\hline & & & & $\mathbf{C}_{\mathbf{T}}$ & $\mathbf{I}_{\mathbf{T}}$ & $\mathbf{H}_{\mathrm{T}}$ \\
\hline 25 & $\begin{array}{l}000000000000000000000000.10000000000000 \\
00000000000000000000000000000000000000 \\
000000000\end{array}$ & 0 & $2.45^{*}$ & 7.93 & 20.77 & 0.24 \\
\hline 31 & $\begin{array}{l}001010000000001100100000011101.11111111 \\
11101100000110000000000000100000000000 \\
000000000\end{array}$ & 2.98 & $3.45^{*}$ & 17.98 & 69.38 & 0.80 \\
\hline 49 & $\begin{array}{l}00000000000000000000000000010000000000 \\
0000000000.0000000000000000000000000000 \\
000000000\end{array}$ & 4.33 & $0.72 *$ & 7.25 & 18.21 & 0.21 \\
\hline 52 & $\begin{array}{l}00010000000000001000100100000010000000 \\
0000100000001.1100000000000000000000000 \\
000000000\end{array}$ & 3.02 & $2.73 *$ & 13.63 & 46.29 & 0.53 \\
\hline 53 & $\begin{array}{l}00000000000000000000000000000000000000 \\
00000000000001.000000000000000000000000 \\
000000000\end{array}$ & 6.09 & $2.64^{*}$ & 8.09 & 21.38 & 0.25 \\
\hline 71 & $\begin{array}{l}00000000000000000000000000000000000000 \\
00000000000000000000000000000000.100001 \\
000000000\end{array}$ & 0 & $2.70 *$ & 9.32 & 26.37 & 0.30 \\
\hline 79 & $\begin{array}{l}00000000001000000000000000000000000000 \\
00000000000000000000000000000000000000 \\
00.0010001\end{array}$ & 3.5 & $1.78^{*}$ & 10.11 & 29.74 & 0.34 \\
\hline
\end{tabular}



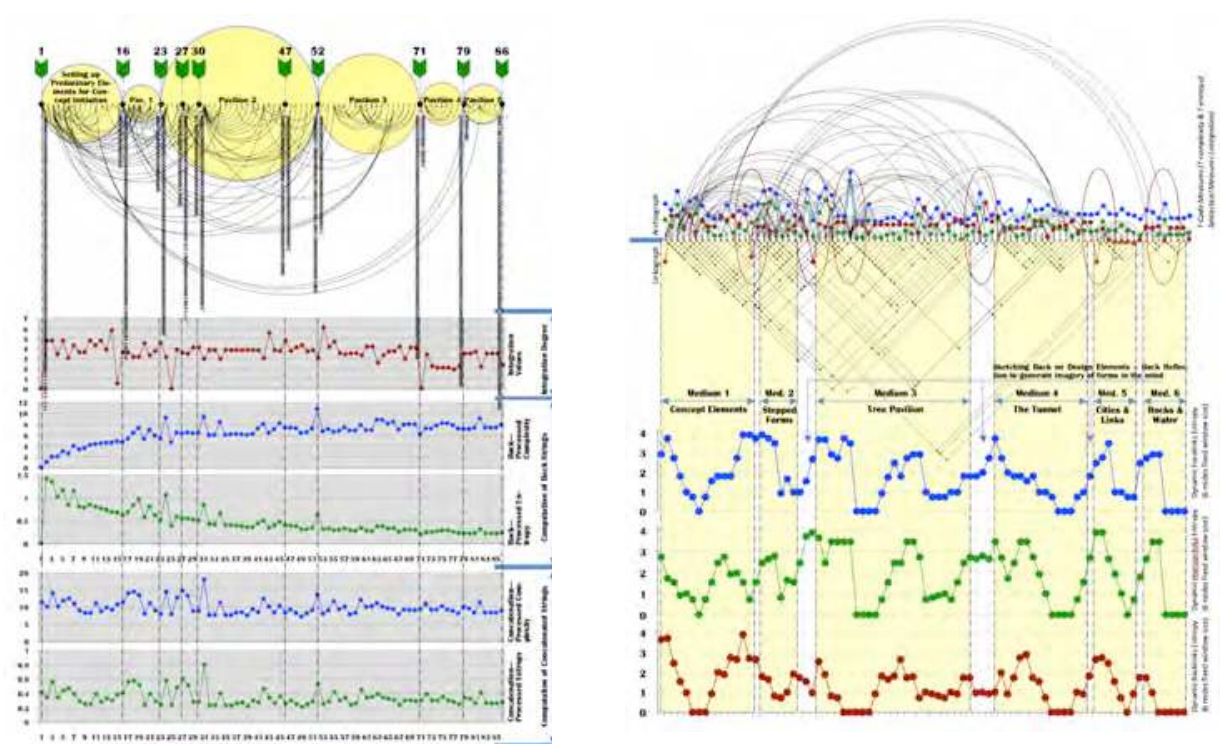

Fig. 11 (a) Overlaying string computation graphs on the archiograph (left) and (b) Overlaying T-code sets, integration, and dynamic entropy (right)

\section{Acknowledgment}

I wish to thank Dr Alkistis-Zoi Skarlatou for her participation in the design experiment providing this study with valuable insights. I also acknowledge the contribution of Dr Ulrich Speidel (Department of Computer Science, University of Auckland, New Zealand) who has provided clear directions on the deterministic information theory and T-code sets. I am grateful for his generous advice and comments. I am also indebted to Consultant Engineer Mohamed Abdallah (Thrupoint, USA) for his thoughtful suggestions and technical support to reach an accurate computational model for this proposition. Finally, my gratitude goes to Prof. Alan Penn (Bartlett School of Graduate Studies, UCL) for his constructive supervision, critical and inspiring throughout and to the school for providing the facilities to establish this research. I take full responsibility for any shortcomings in this paper. 


\section{References}

1. Titchener, M. (1998) 'Deterministic Computation of String Complexity, Information and Entropy'. International Symposium on Information Theory, MIT, Boston, 16-21 August 1998.

2. Titchener, M. (2004) 'The Practical Measurement of Information and Entropy', Online tutorial: http://tcode.auckland.ac.nz/tutorial.html.

3. Titchener, M., Nicolescu, R., Staiger, L., Gulliver, A. and Speidel, U. (2005) 'Deterministic Complexity and Entropy', Fundamenta Informaticae, 64(1-4), pp 443-61.

4. Schön, D.A. (1983) The Reflective Practitioner: How Professionals Think in Action, New York: Harper Collins, Basic Books.

5. Goldschmidt, G. (1991) 'The Dialectics of Sketching', Creativity Research Journal, 4 (2), pp 123-143.

6. Weaver, W and Shannon, C.E. (1949) The Mathematical Theory of Communication, Urbana, Illinois: University of Illinois Press.

7. Kan, J.W.T. and Gero, J.S. (2005) "Can Entropy Indicate the Richness of Idea Generation in Team Designing?" in A. Bhatt (ed.), CAADRIA '05, New Delhi: TVB, Vol. 1, pp. 451-57.

8. Chou S B (2007) 'A Method of Evaluating Creativity In Linkography', 10th QMOD Conference of Quality Management and Organizational Development. Our Dreams of Excellence, 26, Helsingborg, Sweden, July 2007, 18-20.

9. Titchener, M. (2008) "An Introduction to T-codes", Online Tutorial: http://tcode.auckland .ac.nz/ mark/T-codes\%3A\%20Intro.html

10. Speidel, U., Titchener, M.R. and Yang, J. (2006) "How Well Do Practical Information Measures Estimate the Shannon Entropy", CSNDSP, Patras, Greece, 19-21 July 2006.

11. Speidel, U. (2008) "T-code and T-information Theory, Online Tutorial: http://citr.auckland.ac.nz/ ulrich/research.html

12. Tschimmel, K. (2010) "Design as a Perception-in-Action Process", in T. Taura and Y. Nagai (eds.), Design Creativity, London: Springer Verlag, pp. 223-230.

13. Akin Ö. and Akin, C. (1996) 'Frames of Reference in Architectural Design: Analysing the Hyper-acclamation (Aha!)', Design Studies 17 (4), pp 341-361.

14. Goldschmidt, G. (1994) 'On Visual Design Thinking: The Vis Kids of Architecture', Design Studies 15 (2), pp 158-174.

15. El-Khouly, T. and Penn, A. (2012) 'Order, Structure and Disorder in Space Syntax and Linkography: Intelligibility, Entropy, and Complexity Measures', Paper ref. 8248 in M. Green, J. Reyes and A. Castro (eds.). Eighth International Space Syntax Symposium (SSS8), Pontifica Universidad Católica: Santiago De Chile, pp 1-22. 